\title{
Microwave Measurements of Dielectric Properties of Corn Vegetation at C-Band and Comparison with Debye- Cole Dual Dispersion Model
}

\author{
Ashish B. Itolikar ${ }^{\mathrm{a}}$, M.L. Kurtadikar ${ }^{\mathrm{b}}$ \\ ${ }^{a}$ Applied Science Department, Jawaharlal Nehru Engineering College, Aurangabad (MS), India \\ ${ }^{\text {b}}$ P.G. Department of Physics, Faculty of Science, J.E.S. College, Jalna (MS), India \\ *Correspondence: ab001_ito@rediffmail.com
}

\begin{abstract}
Microwave irradiations from vegetation canopy are strongly influenced by its dielectric properties and give useful information for microwave remote sensing -interpretation and applications. This paper reports on the laboratory measurements of dielectric properties of corn vegetation at $\mathrm{C}$-Band frequency. Von Hipple method is used to measure complex dielectric properties using automated C-Band microwave bench set up. The least square fitting technique is used to calculate dielectric constant $\left(\varepsilon^{\prime}\right)$, dielectric loss $\left(\varepsilon^{\prime \prime}\right)$ and errors in their measurements. The measured data is compared with Debye-Cole Dual Dispersion model. Emissivity and radiometric brightness temperature are theoretically estimated from measured dielectric properties at different angles of incidence for dry and moist corn leaves using Fresnel equations.
\end{abstract}

Index Terms - Corn leaves, dielectric constant, microwave remote sensing, Von Hipple method

\section{INTRODUCTION}

Vegetation dielectric properties play key role in microwave remote sensing (MWRS) interpretation and applications. The dielectric property of vegetation canopy is important factor that determines the interaction of canopy with electromagnetic waves, affects microwave (MW) emission and scattering. The complex dielectric property is the ability of material to absorb, reflects and transmits the electromagnetic energy incident and is a measure of electric conductivity. It is ability of material to polarize under the influence of electric field. The complex dielectric property consists of real dielectric constant and imaginary dielectric loss and both are related by following equation number 1 . 
Journal of Microwaves, Optoelectronics and Electromagnetic Applications, Vol. 16, No. 4, December 2017955 DOI: http://dx.doi.org/10.1590/2179-10742017v16i41087

$\epsilon^{*}=\epsilon^{\prime}-\mathrm{j} \epsilon^{\prime \prime}$

Where

$\epsilon^{\prime}-$ Dielectric constant (real part)

$\epsilon "$ - Dielectric loss (imaginary part)

The dielectric properties of vegetation are depend on temperature and salinity dominantly on water content [1].The complex dielectric property of vegetation is combined effect of vegetation constituents i.e. leaves, branch, stem and fruits. Most of the covered part of canopy is leaf. In present work, the laboratory measurement of the complex dielectric properties were conducted for corn leaves at $\mathrm{C}-\mathrm{Band}$ (in this case $5 \mathrm{GHz})$ frequency, at room temperature $\left(27^{0} \mathrm{C}\right)$.

Corn leaves are isohydric in nature (water potential of leaves does not fluctuate during day regardless of soil water status) [2]. The leaf is heterogeneous medium consists of water, bulk organic matter and air spaces. But water in fresh leaf is major (80-90\%) constituent and also important for overall growth of the plant [3]. Therefore the dielectric properties of the leaves are mainly driven by water content. These facts motivate to conduct the dielectric measurements of corn leaves as a function of moisture content.

In this paper laboratory dielectric measurements were performed for freshly cut corn leaves (containing natural moisture) and of different moisture content (due to natural evaporation over time) including dry (oven dried $0 \%$ moisture).This study provides relationship between dielectric property and physical characteristics like moisture of vegetation leaves. The measured data of dielectric constant and loss is compared by Debye-Cole Dual dispersion model constructed on by Ulaby and ElRayes, (1987) [4]. The laboratory validation of dielectric properties is also important for data pertaining to MWRS [5]. Further radiometric emissivity and brightness temperature are estimated from measured dielectric properties at a different angle of incidence for the moist and dry corn leaves by using Fresnel's equations. This data is useful for passive remote sensing of vegetation.

Such type of work was insisted by Indian Space Research Organization (ISRO) in anticipation of launching of MWRS Satellite at C-Band frequency. Dielectric properties of the soil [6], sea water [7] and vegetation like Algae-Aquatic Vegetation [8] were studied and reported by MW group of this laboratory by using the same method and the same C-Band microwave bench set up provided by Brazilian Microwave and Optoelectronics Society-SBMO received 18 Aug 2017; for review 19 Aug 2017 ; accepted 16 Oct 2017 Brazilian Society of Electromagnetism-SBMag 
Indian Space Research Organization (ISRO) under a research project to the PI, (M. L. Kurtadikar) and is used for present work as well.

\section{MATERIALS AND METHOD}

\section{A. Fabrication of sample cell for vegetation leaves}

A solid dielectric cell with a movable reflector for compaction of vegetation leaves was fabricated. It consists of a movable rectangular reflector that is fitted on the rear end of the waveguide cell. The dimensions of the reflector are just smaller than that of the inner dimensions of the wave guide so that it can easily slide inside the wave guide cell. A long screw is connected to the rear side of the solid reflector and is externally connected to a micrometer screw gauge. By rotating the micrometer screw gauge, the thickness of sample inside the cell is measured. Sample is fitted between the reflector and a mica sheet by giving appropriate rotations to the screw for homogeneity and constant compactness of each sample inside the cell. These modifications were carried out at a workshop of the government Industrial Training Institute, Aurangabad (MS), India. The modified sample cell is shown in Fig.1.This cell is already used to measured dielectric properties of other vegetation sample banana reported [9].

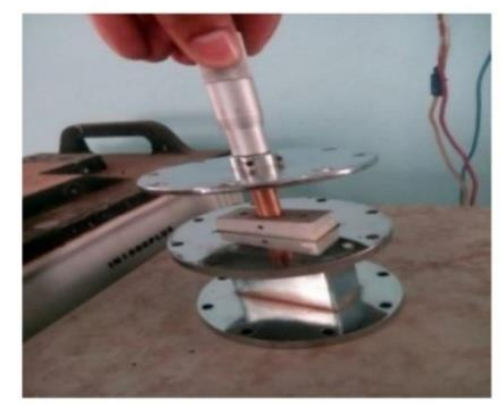

Fig. 1 Sample cell fabricated for vegetation material

\section{B. Sampling}

Leaves of a fresh corn crop were cut from the farm location, 5 kilometres away from Jalna city, Maharashtra state, India. Leaves were cut from various crops of corn having different height (01.0 to $02.2 \mathrm{~m}$ ). The measurements were started on the same day to preserve the physical characteristics of leaves. A freshly cut corn leaf starts shrinking and loses moisture content over a period. To avoid the loss of moisture, the leaves were covered in a polythene bag and transported to the laboratory. The 
leaves were cut in to the rectangular shapes the size of the cross-section of the waveguide cell. Bunches of the cut size leaves were inserted in to the waveguide cell with a compactness to achieve the homogeneity of the material medium. The equal and constant compactness was maintained by fitting the sampled leaves in the cell between the reflector (rear end of the cell) and the mica window (front end of the cell). The reflector is movable and can slide inside the cell to push the sample towards the mica window. Due to time consumption of transportation of sample to laboratory and sampling, tracing of natural moisture is difficult for laboratory measurements. The natural moisture mentioned in this paper is inclusion of this barrier. Authors able to trace $82.51 \%$ moist corn leaves. The dielectric measurements were done for freshly cut corn leaves with natural $(82.51 \%)$, and of different moisture $73.95 \%, 56.06 \%, 16.10 \%$ and $00 \%$ (oven dry). Then the moisture of leaves is reduced by the natural evaporation over a period and finally the sample is oven dried at $70^{\circ} \mathrm{C}$ in oven for 24 hours. The moisture of the leaves was measured gravimetrically on the weight basis given in equation 2.

$\%$ Moisture content $=\frac{\mathrm{Ws}-\mathrm{Wd}}{\mathrm{Ws}} \times 100$

Where,

$\mathrm{W}_{\mathrm{s}^{-}}$Weight of sample

$\mathrm{W}_{\mathrm{d}}$-weight of dry sample

\section{Experimental set up}

There are a several methods of the dielectric measurement [10]. The dielectric of corn leaves was measured using Von Hipple method [11]. It has good accuracy agreement and is easy too. The block diagram of the setup is shown in Fig. 2. It consists of a low power microwave source Varactor Tuned Oscillator (VTO), isolator, coaxial-waveguide adapter, attenuator, Sliding Screw tuner (SS tuner), slotted section and modified solid dielectric cell with movable reflector.

MW generated by the VTO is propagated through passive components of rectangular waveguides into the dielectric cell with a perfect reflector at the closed rear end. The MW source is tuned to give $5 \mathrm{GHz}$ frequency by applying a tuning voltage of 7 volts. An attenuator is used to keep the desired power in the waveguide assembly of the bench. A slotted section with a tuneable probe 
containing a 1N23 detector with the square law characteristics has been used to measure power (current) along the slotted line.

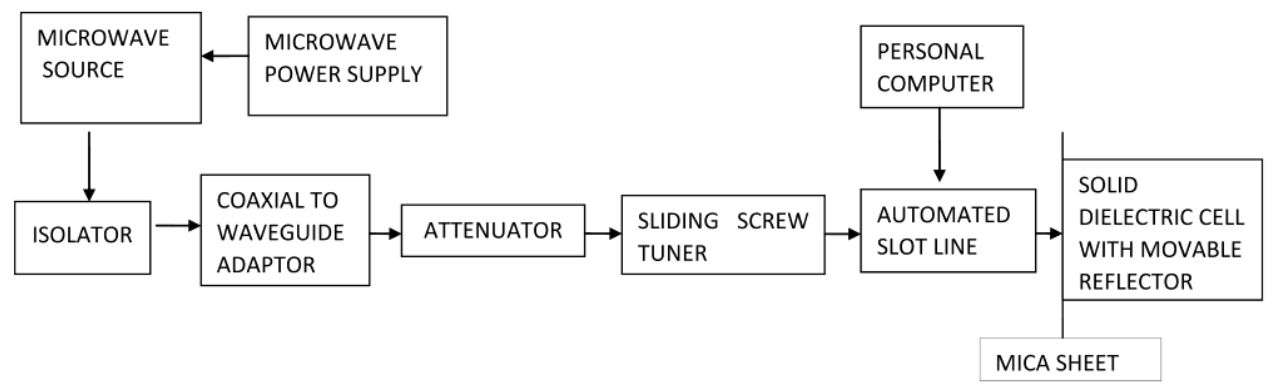

Fig. 2 Block diagram of automated C-Band Microwave bench set up

The detector is connected to a micro ammeter and to the PC to read and record the measured power. The probe sits on the slot line such that the tip of the tuneable probe is slightly penetrated and it can be moved forwarded and backward along the slot line section to sense the electromagnetic field in the wave guide. The bench is tuned to get a symmetrical standing wave pattern in the slot line. The tuning of the bench is possible by critical adjustments of the probe detector and S.S. Tuner. A symmetrical standing wave pattern with an empty dielectric cell is obtained. The dielectric sample under consideration (corn leaves) is inserted in the dielectric cell with a constant compaction (for homogeneity of the medium). The probe is traversed along the slot line at equal intervals and power (current) is recorded with respect to the corresponding probe positions. This data is acquired and stored in a file using a microcontroller interface system. This data makes use of $\alpha$ and $\beta$ as fitting parameters, where $\alpha=$ attenuation factor, $\beta=$ phase shift constant. The data is stored for corn leaves samples of different thicknesses (in this case for three thicknesses $0.5 \mathrm{~cm}, 1.0 \mathrm{~cm}$ and $1.5 \mathrm{~cm}$ ). The guided wavelength $\lambda_{\mathrm{g}}$ is measured from the minima of the standing wave pattern.

$\beta=\frac{2 \pi}{\lambda_{\mathrm{g}}}$

The free space wavelength, $\lambda_{0}$ is determined using the relation refer equation 4 .

$\frac{1}{\lambda_{0}^{2}}=\frac{1}{\lambda_{\mathrm{g}}^{2}}+\frac{1}{\lambda_{\mathrm{c}}^{2}}$ 
Where, $\lambda \mathrm{c}=2 \times \mathrm{a}=2 \times 4.73=9.46 \mathrm{~cm}$, 'a' being the broader side of the C-band rectangular waveguide. The real and imaginary parts of the complex dielectric constant are calculated using the relations given in equations 5 and 6 .

$$
\begin{aligned}
& \varepsilon^{\prime}=\lambda_{0}^{2}\left(\frac{1}{\lambda_{\mathrm{c}}^{2}}+\frac{\left(\alpha^{2}-\beta^{2}\right)}{4 \pi^{2}}\right) \\
& \varepsilon^{\prime \prime}=\frac{\lambda_{0}^{2} \alpha \beta}{2 \pi^{2}}
\end{aligned}
$$

A source code for computing a dielectric constant has been developed. The number of data files, for different thickness of the samples are combined to get a single input data file, which can be used, in the source code for calculating dielectric constant $\left(\varepsilon^{\prime}\right)$ and loss $\left(\varepsilon^{\prime \prime}\right)$ with errors in the measurements as $\Delta \varepsilon^{\prime}$ and $\Delta \varepsilon^{\prime \prime}$. For errors in measurements refer Table 1 .

Least square fitting technique is used to compute dielectric properties and errors. The experimental data makes use of $\alpha$ and $\beta$ as fitting parameters, where $\alpha=$ attenuation factor, $\beta=$ phase shift constant.

When the source code is run for computation of the dielectric properties using "inp.dat" as input file, multi valued solutions are obtained for different values of the coefficient of propagation constant "AP (2)" corresponds to a difference factor. The difference factor referred as the difference between the experimental and theoretical calculations. From obtained multiple solutions, those values of AP (2) factor for which the difference factor is minimum are considered. Minimum of all such minima is considered for final calculations (optimum solution) of dielectric constant $\varepsilon^{\prime}$ and the dielectric loss $\varepsilon^{\prime \prime}$ of the sample and the errors $\Delta \varepsilon^{\prime}$ and $\Delta \varepsilon^{\prime \prime}$.

TABLE I. ERRORS IN MEASUREMENTS

\begin{tabular}{|c|c|c|}
\hline $\begin{array}{c}\% \text { Gravimetric } \\
\text { Moisture }\end{array}$ & $\begin{array}{c}\text { Error in Dielectric } \\
\text { Constant }\left(\Delta \varepsilon^{\prime}\right)\end{array}$ & $\begin{array}{c}\text { Error in } \\
\text { Dielectric Loss } \\
\left(\Delta \varepsilon^{\prime}\right)\end{array}$ \\
\hline 82.51 (Natural) & $2.46 \mathrm{E}-02$ & $4.85 \mathrm{E}-02$ \\
\hline 73.95 & $5.88 \mathrm{E}-02$ & $4.85 \mathrm{E}-02$ \\
\hline 56.06 & $5.30 \mathrm{E}-02$ & $5.43 \mathrm{E}-02$ \\
\hline 16.1 & $7.97 \mathrm{E}-02$ & $5.95 \mathrm{E}-02$ \\
\hline 0 (Dry) & $1.63 \mathrm{E}-02$ & $1.62 \mathrm{E}-02$ \\
\hline
\end{tabular}


Journal of Microwaves, Optoelectronics and Electromagnetic Applications, Vol. 16, No. 4, December 2017960 DOI: http://dx.doi.org/10.1590/2179-10742017v16i41087

D. Debye Cole Dual Dispersion Model (Ulaby\& El-Rays, 1987)

This model is chosen because it has physical basis for formulation. The complex dielectric constant of leaf is modelled as a simple mixture of three components:

(i) Non dispersive residual component, $\varepsilon_{\mathrm{s}}$;

(ii) a free water component $\mathrm{v}_{\mathrm{fw}} \varepsilon_{\mathrm{w}}$, where $\mathrm{v}_{\mathrm{fw}}$ is volume fraction of water and $\varepsilon_{\mathrm{w}}$ the free water dielectric constant; and

(iii) A bulk vegetation bound water component $v_{b} \varepsilon_{b}$, where $v_{b}$ is volume fraction of bulk vegetation-bound water component and $\varepsilon_{\mathrm{b}}$ its dielectric constant.

$\varepsilon=\varepsilon_{s}+v_{f w} \varepsilon_{w}+v_{b} \varepsilon_{b}$

The values of $\epsilon_{\mathrm{w}}$ can be estimated using Debye Equation. Substituting the equation for $\epsilon_{\mathrm{w}}$ and $\epsilon_{\mathrm{b}}$ in equation 7 can be written as.

$\varepsilon=\varepsilon_{s}+V_{f w}\left[4.9+\frac{73.5}{1+\frac{j f}{19.7}}-j \frac{18 \sigma}{f}\right]+V_{b}\left[2.9+\frac{55.0}{\left(1+\frac{j f}{0.18}\right)^{0.5}}\right]$

$\varepsilon_{s}=1.7-0.74 \mathrm{M}_{\mathrm{g}}+6.16 \mathrm{M}_{\mathrm{g}}^{2}$

$V_{f w}=M_{g}(0.55 M g-0.076)$

$V_{b}=\frac{4.64 M_{g}^{2}}{1+7.36 M_{g}^{2}}$

$\sigma=1.27$

Where,

$\mathrm{J} 2=-1$, f- Frequency in $\mathrm{GHz}, \mathrm{Mg}$ - Gravimetric moisture content and $\sigma$ - Ionic conductivity.

E. Estimation of Emissivity and Brightness Temperature

From the definition of emissivity can be expressed as given in equation 13

$e_{s(p)}=\left(1-R_{s(p)}\right)$

Where $\operatorname{Rs}(\mathrm{p})$ is the smooth- surface reflectivity. For a homogeneous leaves sample with a smooth surface, the reflectivity at vertical and horizontal polarizations, $\mathrm{RsV}$ and $\mathrm{RsH}$, are given by the Fresnel expressions given by equations 14 and 15 [12].

$R_{s V}=\left|\frac{K \cos u-\sqrt{K-\sin ^{2} u}}{K \cos u+\sqrt{K-\sin ^{2} u}}\right|^{2}$ 
Journal of Microwaves, Optoelectronics and Electromagnetic Applications, Vol. 16, No. 4, December 2017961 DOI: http://dx.doi.org/10.1590/2179-10742017v16i41087

$\mathrm{R}_{\mathrm{sH}}=\left|\frac{\cos \mathrm{u}-\sqrt{\mathrm{K}-\sin ^{2} \mathrm{u}}}{\cos \mathrm{u}+\sqrt{\mathrm{K}-\sin ^{2} \mathrm{u}}}\right|^{2}$

Where ' $\mathrm{u}$ ' is the incidence angle and ' $\mathrm{K}$ ' is the absolute value of the dielectric constant of corn leaves, which is a measure of the response of the leaves to an electromagnetic wave and is largely determined by moisture content of the leaves. Emissivity of corn leaves for different moisture content, for different angles of incidence is calculated using Fresnel equations.

Passive microwave remote sensing is based on the measurement of thermal radiation in the centimetre wave band of the electromagnetic spectrum $\mathrm{T}_{\mathrm{b}}$. This radiation is determined largely by the physical temperature and the emissivity of the radiating body and can be approximated by using equation 16.

$\mathrm{T}_{\mathrm{b}(\mathrm{p})} \approx \mathrm{e}_{\mathrm{s}(\mathrm{p})} \mathrm{T}$

Where $T_{b}$ observed brightness temperature; $T$ physical temperature of the emitting layer; $p$ refers to vertical or horizontal polarization; $\mathrm{e}_{\mathrm{s}}$ - smooth-surface emissivity.

\section{RESULTS AND DISCUSSION}

Fig. 3 and 4 shows the variation of $\varepsilon^{\prime}$ and $\varepsilon^{\prime \prime}$ as a function of gravimetric moisture content of corn leaves respectively. It is observed that both the measured $\varepsilon^{\prime}$ and $\varepsilon^{\prime \prime}$ are increased exponentially with percent gravimetric moisture content. For low moistures, $\varepsilon^{\prime}$ and $\varepsilon^{\prime \prime}$ increased with slow rate. However the rate is more rapid for high moist leaves. This might be due to the polarizability of free and bound water molecules. When electric field at MW frequency is interacted with asymmetric molecule like water linked with bulk material. It gets polarized in dipolar manner. At low moisture the water molecules are tightly bound to the bulk material of leaves causes less polarization. As moisture increased further the water molecule becomes free and loosely bound to the system causes more polarization.

The measured data of $\varepsilon^{\prime}$ of corn leaves has good agreement with model values. This agreement may be due to the fact that the original model was constructed by using the corn vegetation. The measured $\varepsilon^{\prime \prime}$ is not agreeable with the model values. The difference of measured and predicted $\varepsilon^{\prime \prime}$ may contributes due to the difference in measurement method (The actual model is 
Journal of Microwaves, Optoelectronics and Electromagnetic Applications, Vol. 16, No. 4, December 2017962 DOI: http://dx.doi.org/10.1590/2179-10742017v16i41087

constructed by considering the measurements using coaxial probe technique whereas present measurements are using slot line technique) or the errors in the measurement of loss. In slot line technique the accuracy of measuring the real part was within $10 \%$, while for the imaginary part sizable errors were reported (Broadhurst,1970). The possible source of errors associated with slot line is accuracy of standing wave ratio measurement, variation of maxima and minima at different points on the line referred as slope error which is caused by variation of probe depth, probe tuning errors due to excessive probe penetration to the electromagnetic field in slot line etc. [13].

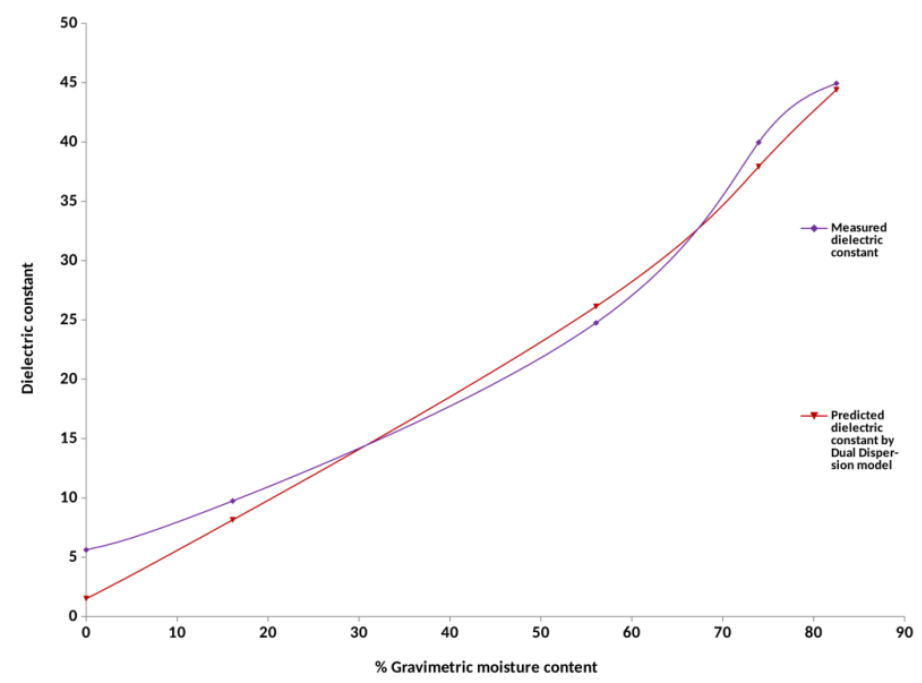

Fig. 3 Dielectric constant $\left(\varepsilon^{\prime}\right)$ of corn leaves as a function of \% gravimetric moisture content

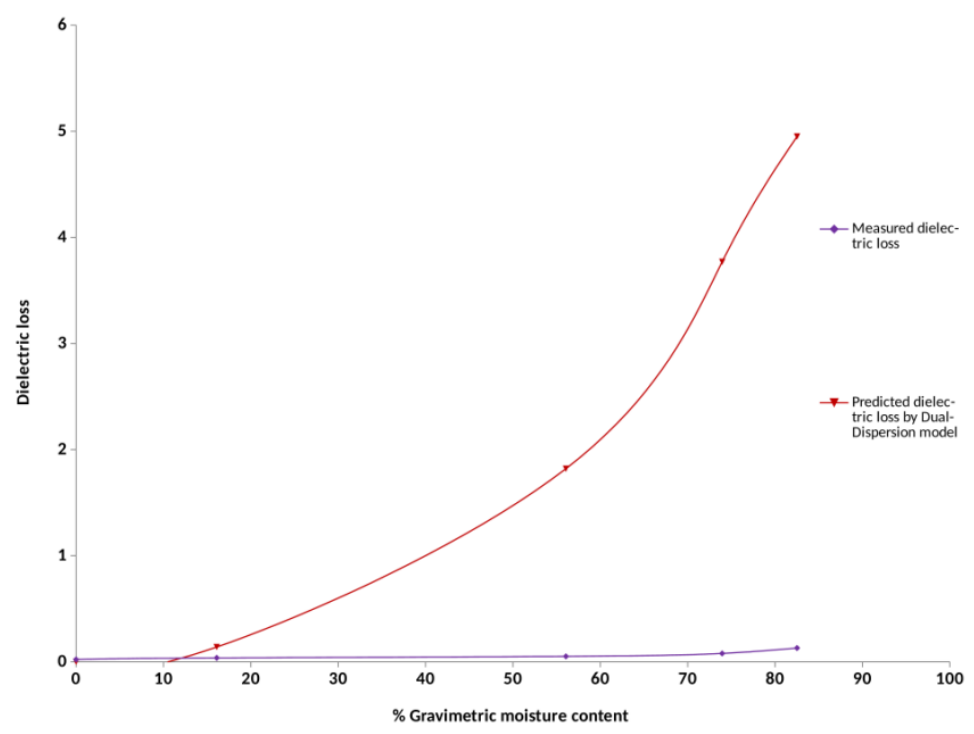

Fig. 4 Dielectric loss $\left(\varepsilon^{\prime \prime}\right)$ of corn leaves as a function of $\%$ gravimetric moisture content 
It leads to conduct more dielectric measurements with different vegetation species to validate this model. The emissivity is estimated theoretically from measured dielectric properties at different angle of incidence for dry and moist corn leaves as shown in Fig.5. At normal incidence the emissivity is ranges from 0.83 (for $0 \%$ moist leaves) to 0.45 (for 85.51 moist leaves) and is decreases as moisture increase.

The curves for horizontal polarization show a decrease of emissivity with increase in angle of incidence for the corn leaves. The rate of decrease of emissivity value is very slow initially up to $20^{\circ}$, after then it is comparatively rapid. The curves for vertical polarization show increase of emissivity initially at slow rate and after angle of incidence is $25^{\circ}$, the rate become more up to angle of incidence from $70^{\circ}$ to $85^{\circ}$ beyond which the emissivity trend for vertical polarization is reversed and found to be decreasing.

This change of emissivity in range of angle of incidence from $70^{\circ}$ to $85^{\circ}$ varies with moisture content of the corn leaves. Fig.6 shows brightness temperature at different angle of incidence for moist and dry corn leaves. It ranges from 252 (for $0 \%$ moist) to 136 (for $81.51 \%$ moist) and is decreases as moisture is increased. The trends of brightness temperature for horizontal and vertical polarization are same as that of emissivity for corn leaves.

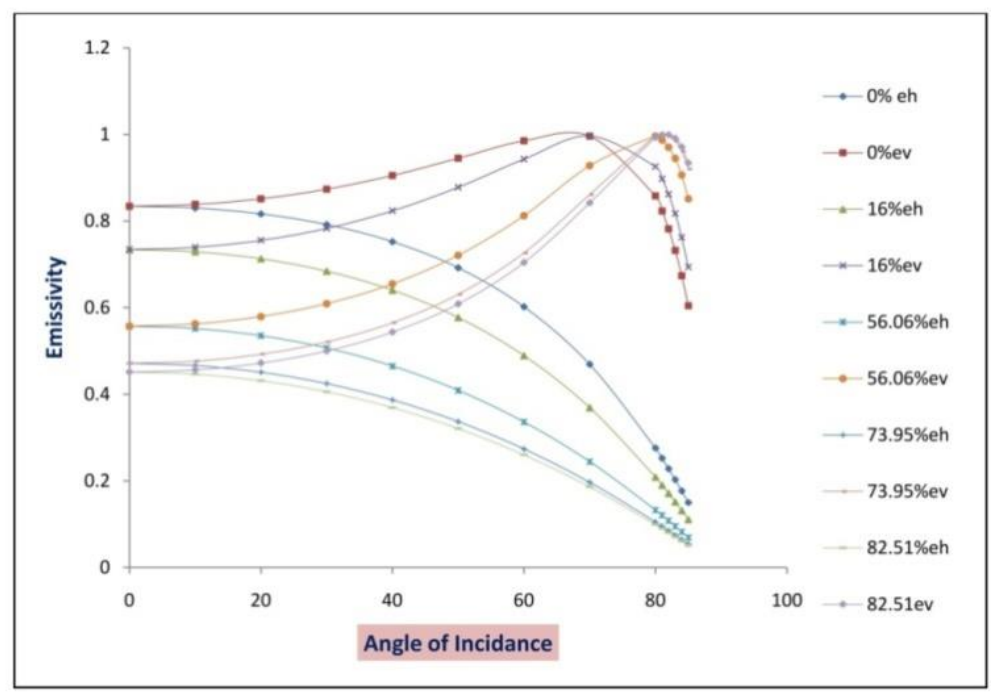

Fig. 5 Emissivity at different angle of incidence of dry, 16\%, 56.06\%, 73.95\% and $82.51 \%$ moistures of corn leaves for horizontal and vertical polarization 


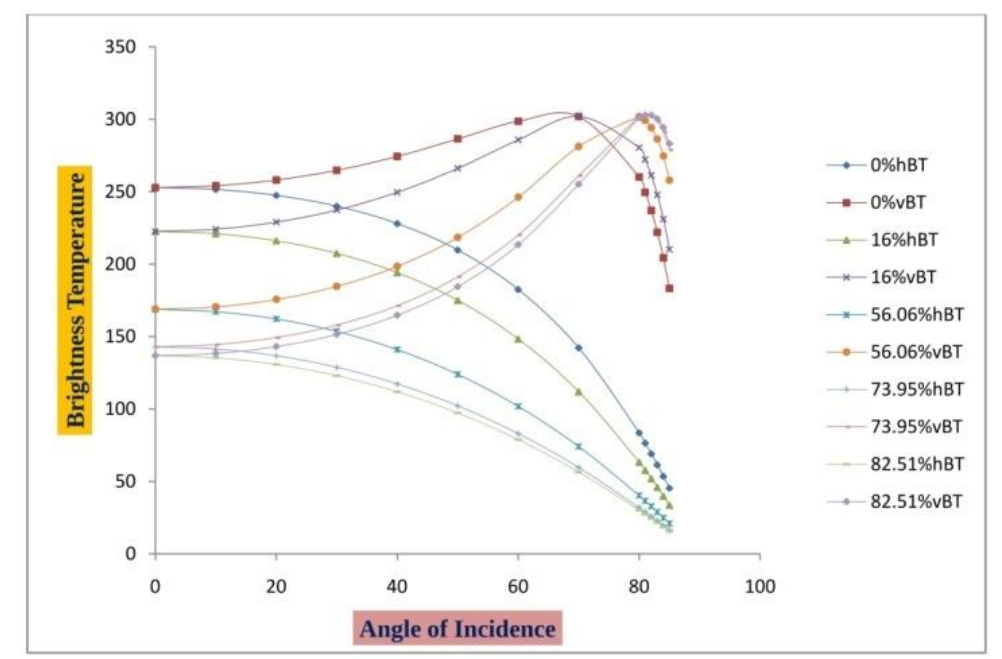

Fig.6 Brightness temperature at different angle of incidence of dry, 16\%, 56.06, 73.95, 82.51\% moistures of corn leaves for horizontal and vertical polarization

\section{CONCLUSIONS}

The present investigations provide the experimental results of dielectric properties as a function of gravimetric moisture at C-Band frequency for corn vegetation. The relationship is direct between dielectric properties and physical characteristics (in this case moisture). This linkage is important and provide physical basis for MWRS of vegetation. Estimated emissivity and brightness temperature are the parameters that can be used for designing passive MW sensors especially for vegetation assessment. The comparison of measured dielectric properties with theoretical model (Ulaby and ElRays, 1987) implies that the model performance is good for just prediction of dielectric constant only and not for dielectric loss of vegetation. This work provides the dielectric data base for corn vegetation in Indian context.

\section{ACKNOWLEDGMENT}

Authors are thankful to Indian Space Research Organization (ISRO) for providing the facility of MW C-Band set up through "RESPOND" project at J.E.S. College Jalna. Prof. S.C. Mehrotra, Ramanujan Chair of Remote Sensing and GIS, is also acknowledged for fruitful discussion.

\section{REFERENCES}

[1] Nelson, Stuart O. IEEE Trans. on Electrical Insulation. 1991 26(5), 845-869

[2] Francois, Tardieu; Thierry, Simonneau. Journal of Experimental Botany. 1998 49, 419-432

[3] Ferguson, Marvin H. USGA Journal and Turf management. 1959, 30-33.

[4] Fawwaz T, Ulaby; Mohamed A, EL-Rayes. IEEE Trans.on Geo. and Remote Sensing, 1987,GE-25(5) 550-557

[5] Kurtadikar, M.L.; Popalghat, S.K.; Mehrotra, S.C.2013, International Experts Meet on Microwave Remote Sensing, Abstract-2, 16-17

[6] Deshpande, S.S.;Itolikar, A.B.;Joshi,A.S.,Kurtadikar,M.L. In Conference Proceedings: 11th International Conference on Microwaves, Antena, Propagation \& Remote Sensing ICMAR-2015,India, Dec.15-17,2015. 
Journal of Microwaves, Optoelectronics and Electromagnetic Applications, Vol. 16, No. 4, December 2017965 DOI: http://dx.doi.org/10.1590/2179-10742017v16i41087

[7] Joshi, A.S.; Kurtadikar, M.L. Journal of Geomatics SAC-ISRO- The Journal of Indian society of Geomatics, 2013, 7(1), $33-40$

[8] Itolikar, Ashish B.; Kurtadikar, M.L. Bionano Frontier, 2015 8(3), 371- 374.

[9] Itolikar, Ashish B.; Kurtadikar, M.L. Int. Journal of Advances in Remote Sensing and GIS, 2017, Vol.5, No.1, 58-66

[10] Kaatze, Udo. IOP publishing, Metrologia, 2010, 47, 91-113

[11] Von Hipple, A.R., Dielectric materials and Applications, New York, Wiley-1954.

[12] Griffiths, David J., Introduction to Electrodynamics, Prentice Hall, ISBN 0-13-805326-X, 390

[13] Kulkarni,P.G., Ph.D. Thesis submitted to Dr. Babasaheb Ambedkar Marathawada University,Aurangabad, India, 2006. 